\title{
APPLICATIONS OF ACTION CAM SENSORS IN THE ARCHAEOLOGICAL YARD
}

\author{
M. Pepe ${ }^{1 *}$, S. Ackermann ${ }^{2}$, L. Fregonese ${ }^{2}$, F. Fassi ${ }^{2}$, A. Adami ${ }^{2}$ \\ ${ }^{1}$ University of Naples "Parthenope", 80143 Naples, Italy \\ massimiliano.pepe@uniparthenope.it \\ ${ }^{2}$ Polytechnic of Milan, 20133, Milan, Italy \\ (sebastiano.ackermann, luigi.fregonese, francesco.fassi, andrea.adami)@ polimi.it
}

Commission II, WG II/8

KEY WORDS: Action camera; Point clouds; Go-pro; Blur; Chromatic aberration, Archaeological yard.

\begin{abstract}
:
In recent years, special digital cameras called "action camera" or "action cam", have become popular due to their low price, smallness, lightness, strength and capacity to make videos and photos even in extreme environment surrounding condition. Indeed, these particular cameras have been designed mainly to capture sport actions and work even in case of dirt, bumps, or underwater and at different external temperatures. High resolution of Digital single-lens reflex (DSLR) cameras are usually preferred to be employed in photogrammetric field. Indeed, beyond the sensor resolution, the combination of such cameras with fixed lens with low distortion are preferred to perform accurate 3D measurements; at the contrary, action cameras have small and wide-angle lens, with a lower performance in terms of sensor resolution, lens quality and distortions. However, by considering the characteristics of the action cameras to acquire under conditions that may result difficult for standard DSLR cameras and because of their lower price, these could be taken into consideration as a possible and interesting approach during archaeological excavation activities to document the state of the places.

In this paper, the influence of lens radial distortion and chromatic aberration on this type of cameras in self-calibration mode and an evaluation of their application in the field of Cultural Heritage will be investigated and discussed. Using a suitable technique, it has been possible to improve the accuracy of the 3D model obtained by action cam images. Case studies show the quality and the utility of the use of this type of sensor in the survey of archaeological artefacts.
\end{abstract}

\section{INTRODUCTION}

Structure from Motion (SfM) is a well-known technique that allows to obtain 3D spatial information about an object using a dataset of 2-D images (Styliandis et. al., 2003; Westoby et al., 2012). It has proved to be among the techniques most used and accurate for the reconstruction of three-dimensional documentation of complex buildings and sites (Jordà et al, 2011). The versatility of this technique allows the acquisition of 3D-data of objects of different size, from a very small machine part up to large buildings (Koch et al., 2009) as well as the application on several fields such as cultural heritage, reverse engineering, archaeology, architecture 3D, documentation and mapping (Menna and Troisi, 2010; Kersten and Lindstaedt, 2012; Remondino et al., 2012; Fassi et al., 2013; Pepe et al., 2018).

In this environments, many studies are in progress using a variety of devices and interest on potential of the action cameras is increasingly the object of investigation.

Balletti et al. (2014) discussed different camera calibration methods using GoPro Hero 3 camera for 3D measurement purposes. In addition, the authors have developed a special software able to perform both the calibration and the production of undistorted scenes for each one of the images and video capturing mode of the sensor tested.
Kim et al., (2014) construct the 3D point clouds of building façades by using super-view stereo video obtained by employing a GoPro HERO 3+ Black Edition sensor.

Teo (2015) compare the image-mode and video-mode of multiple action cameras for $3 \mathrm{D}$ point clouds generation. The camera used for the test was a GoPro Hero 4 Black Edition which is able to collect digital images up to $12 \mathrm{Mp}(4000 \mathrm{x}$ $3000)$ resolution and video up to $8.3 \mathrm{MP}$ (3840 x 2160) resolution at 30 frames per second.

In Hastedt et al. (2016), different calibration procedures for fish-eye lenses are evaluated in order to quantify their accuracy potential in UAV photogrammetry: in this research, GoPro Hero4 Black sensor was used for the experimentation.

The wide diffusion of this products has introduced on the market low-cost sensors, such as that used in the paper of Jurjević and Gašparović (2017).

Using these type of sensors, in each paper above mentioned a special focus on the image processing procedure is discussed in order to overcome the large distortions created by these types of cameras. As regards the methods of calibration, a widely method used in the photogrammetric field is the so-called "selfcalibration": it utilizes the acquired images in order to determine both the calibration parameters that the exterior parameters for the reconstruction of the 3D model (Brown,1971; Fraser, 1997; Pepe and Parente, 2017).

* massimiliano.pepe@uniparthenope.it 
This methodology allows to accelerate the images alignment and the 3D point clouds building process as well as to increase the model accuracy of the acquired object. Over the years, several tests and studies have demonstrated that the result quality of the self-calibration is related to the geometry of the camera network: indeed, a weak geometry of the network and/or a lack of ground control points could provide low accuracy results and reliability (Remondino and Fraser, 2006).

In the present paper, the first section called "Investigations on the technical properties of the action cameras" discusses the images propriety generated from action cam sensors for photogrammetry purpose. The sub-section "Camera calibration parameters" concerns the determination of the internal orientation parameters, (i.e. the focal length $f$, principal point image coordinates $x p$, $y p$, radial and tangential lens distortion coefficients $K 1, K 2, K 3, P 1, P 2$ ), the analysis of the geometric distortions and chromatic aberrations, in order to understand limits and potentials of these cameras in field testing. In addition, in the sub-section "Camera calibration parameters" the characteristics of the sensor tested (internal parameters orientation) are discussed. Subsequently, in the sub-section called "Self-Calibration with a 3D test field", the sensor parameters are investigated by a suitable 3D test field in selfcalibration mode. The sub-section "Chromatic aberration" intends to verify the quality of the images and to evaluate the effect of the chromatic aberration generated by the sensor using a specific chart. The section "Application of action cameras in archaeological field" describe, by three case studies in archaeological environment, a suitable method in order to improve the quality of the 3D model.

\section{INVESTIGATIONS ON THE TECHNICAL PROPERTIES OF THE ACTION CAMERA}

\subsection{Camera calibration parameters}

Action cameras are mainly designed to capture sport actions and obtain videos with immersive experience effects. For this reason, they are usually designed to provide a field of view (FOV) similar to the human one (about $160^{\circ}$ horizontally and $120^{\circ}$ vertically) and equipped with wide angle fixed lens, with a short focal length of about $3 \mathrm{~mm}$. A report of some action cameras available on the market, is reported in Table 1 .

\begin{tabular}{|l|c|c|}
\hline Model & focal length & Resolution \\
\hline Polaroid Cube & $\mathrm{mm}$ & $\mathrm{Mpx}$ \\
GoPro Hero3 Silver & 3,4 & 6 \\
GoPro Hero4 Silver & 2,5 & 11 \\
Sony action cam HDR-AS30V & 5 & 12 \\
Panasonic HX-A1ME-K & 2,5 & 11,9 \\
Sport Trevi go 2200 HD & 2,1 & 3,54 \\
Ricoh WG-M1 & 3 & 1,3 \\
\hline
\end{tabular}

Table 1. Comparison of the focal length and resolution of some typical action camera.

The camera used for the present case study is a generic and cheap "1080p HD Sport Camera" with a 1.3 Megapixel CMOS sensor, equipped with a wide angle lens (FOV of $170^{\circ}$ ) and a focal length of $2.99 \mathrm{~mm}$.

Of course, due to the high FOV of this camera, the radial distortion effect is particularly amplified, and it is a not negligible aspect for photogrammetric purposes: by overlaying a regular grid on a photo of a chessboard captured with the used camera, it can be visually verified the high level of distortion, but also the effect of defocusing that increase by distancing from the centre (Figure 1).

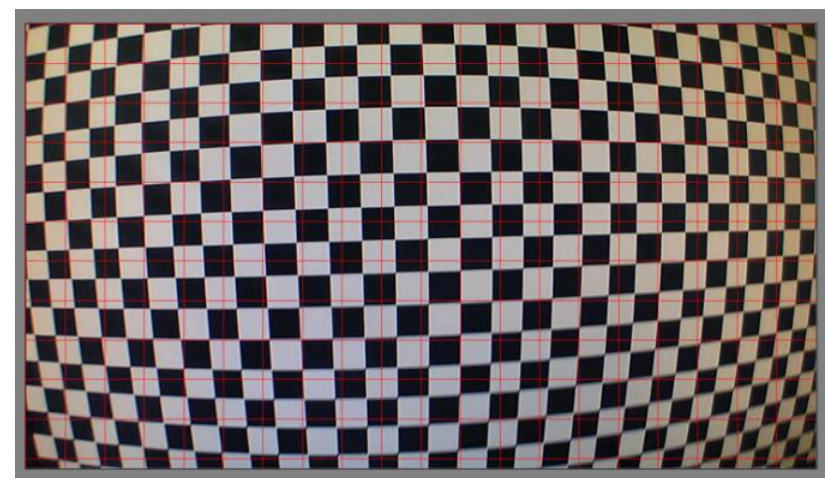

Figure 1. Effect of the image distortion of a chessboard on a regular grid (indicated by the red lines)

Due to the presence of such accentuate distortions, the images that come out with such cameras are distant from the geometrical and mathematical model of the central perspective. In order to better understand the difference from the two models, it is necessary to investigate the intrinsic characteristics of the camera by determining its geometric characteristics $(f, x p$, $\left.y p, K_{1}, K_{2}, K_{3}, P_{1}, P_{2}\right)$.

The software used for the camera calibration is Agisoft Lens (Agisoft, 2017), an automatic lens calibration software that supports estimation of the full camera calibration matrix, including non-linear distortion coefficients. The Brown's model is implemented in the software which it uses the following equations (Brown, 1971):

$$
\left\{\begin{array}{l}
\bar{x}=x+\frac{x}{r}\left(K_{1} r^{3}+K_{2} r^{5}+K_{3} r^{7}\right)+P_{1}\left(r^{2}+2 x^{2}\right)+2 P_{2} x y \\
\bar{y}=y+\frac{y}{r}\left(K_{1} r^{3}+K_{2} r^{5}+K_{3} r^{7}\right)+P_{2}\left(r^{2}+2 y^{2}\right)+2 P_{1} x y
\end{array}\right.
$$

where, $r=\sqrt{x^{2}+y^{2}}, x$ and $\mathrm{y}$ are the measured image coordinates while $\bar{x}$ and $\bar{y}$ are the corrected image coordinates.

\begin{tabular}{|l|c|}
\hline Parameters & Values \\
\hline & pixel \\
$f x$ & $1041.09 \pm 2.26407$ \\
$f y$ & $1041.74 \pm 2.21275$ \\
$c x$ & $587.544 \pm 0.589765$ \\
$c y$ & $358.709 \pm 0.593307$ \\
skew & $0.163291 \pm 0.0949115$ \\
$K_{1}$ & $-0.458073 \pm 0.00319641$ \\
$K_{2}$ & $0.302831 \pm 0.0107819$ \\
$K_{3}$ & $-0.172161 \pm 0.0169433$ \\
$P_{1}$ & $0.000421565 \pm 0.000161426$ \\
$P_{2}$ & $0.000446297 \pm 0.000131516$ \\
\hline
\end{tabular}

Table 2. Action camera calibration parameters.

In the Table 2, the outcome of the camera self-calibration performed with the Agisoft Lens software is reported: horizontal and vertical focal lengths are expressed in pixels.

The curves representing the radial and tangential distortion are reported in Figure 2: of course, the radial distortion curve goes upward to indicate that the lens has a barrel distortion type, and it sensibly increases its trend with respect to the radius, due to the wide angle lens of this camera. 
In order to understand numerically such distortions, a comparison with a DSLR Canon EOS 5D Mark II camera, equipped with a $24 \mathrm{~mm} \mathrm{f} / 2.8$ lens was also performed with the same calibration procedure. The distortion values with a radius of 2500 pixels is equal to 50 pixels, while the same distortion value is obtained at radius value of about 500 pixels in the case of the sport camera.
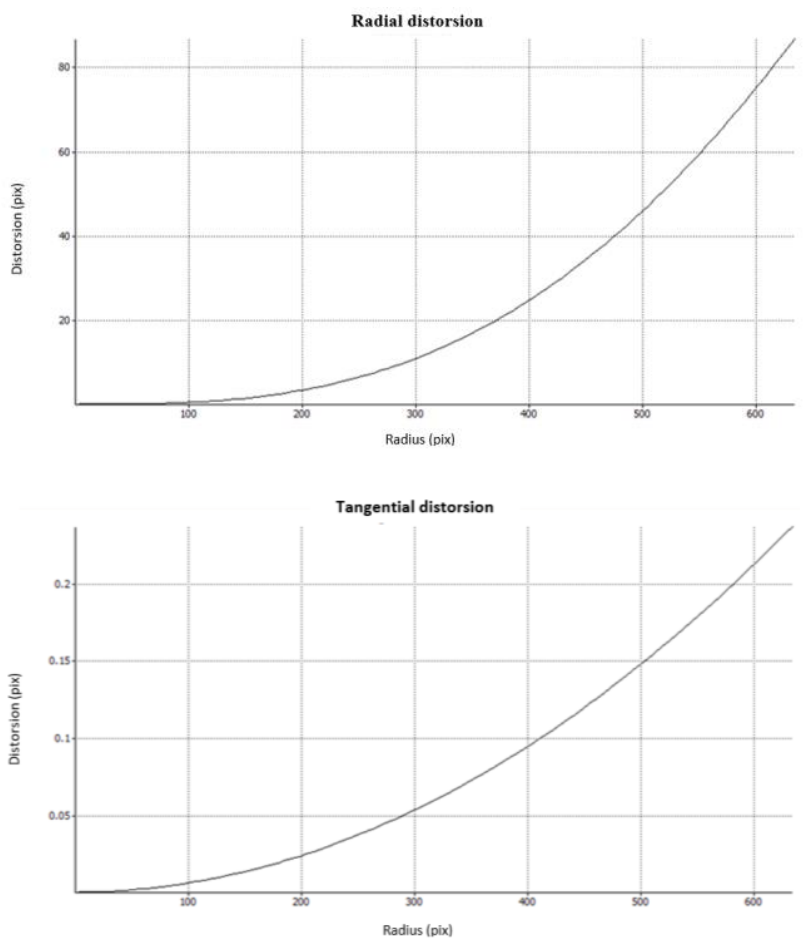

Figure 2. Radial (above) and tangential (below) distortion curves

\subsection{Self-Calibration with a 3D test field}

In order to evaluate the accuracy that can be obtained with the under exam action cam as well as to verify the coherence of the interior parameter obtained with the $2 \mathrm{D}$ test field used in the previous procedure, a second calibration session by using a $3 \mathrm{D}$ test field has been also performed. The 3D test field created for this calibration session was composed by 20 circular coded targets positioned at different heights from the ground. The coordinates of each target within an arbitrary coordinate system were determined by measuring them with a Leica TCRA1103+ total station. An example of representation of camera network is shown in the Figure 3.

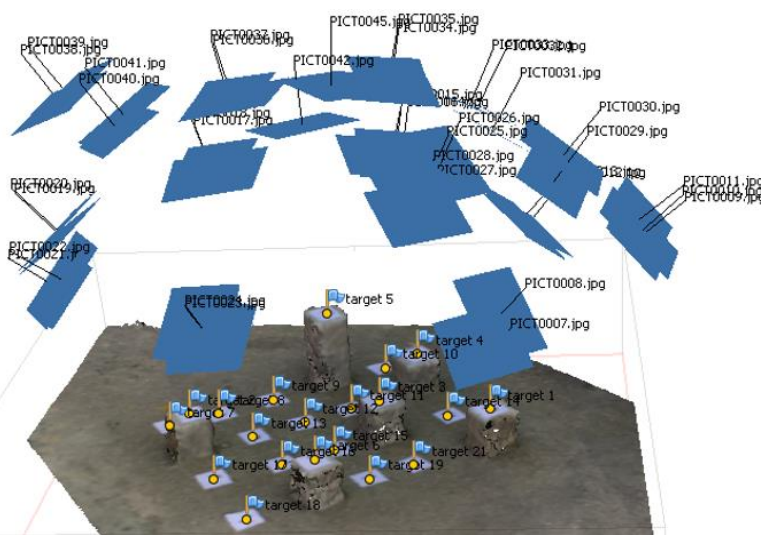

Figure 3. Camera network layout for the tested action camera
The same calibration session was performed with the DSLR Canon EOS 5D Mark II camera equipped with a $24 \mathrm{~mm} \mathrm{f} / 2.8$ lens and with a GoPro Hero3 action camera. For each of the involved cameras, several photos with different viewpoints and orientations were taken in order to obtain an enough robust network camera. The average values of the standard deviations on the GCPs involved are reported in Table 3.

\begin{tabular}{|c|c|c|c|}
\hline \multirow{2}{*}{ Type of camera } & \multirow{2}{*}{$\begin{array}{l}\text { No. } \\
\text { Photos }\end{array}$} & \multicolumn{2}{|c|}{$\begin{array}{c}\text { Calibration Method } \\
\text { Average RMS }\end{array}$} \\
\hline & & $\begin{array}{c}\text { Pre } \\
\text { calibrated }\end{array}$ & $\begin{array}{c}\text { Self } \\
\text { calibration }\end{array}$ \\
\hline & & $\mathrm{mm}$ & $\mathrm{mm}$ \\
\hline $\begin{array}{l}\text { Canon EOS 5D Mark II } \\
\text { - EF-S 24mm }\end{array}$ & 39 & 0.516 & 0.345 \\
\hline $\begin{array}{l}\text { 1080p HD sport camera } \\
\mathrm{f}=2,99 \mathrm{~mm}\end{array}$ & 37 & 0.774 & 0.430 \\
\hline $\begin{array}{l}\text { Go Pro Hero3 } \\
\mathrm{f}=2,5 \mathrm{~mm}\end{array}$ & 56 & 0.835 & 0.729 \\
\hline
\end{tabular}

Table 3. Accuracy on targets; values of average RMS obtained with the several cameras

The results reported in Table 3 highlight that excellent results in terms of accuracy can be reached even with such kind of wide angle cameras. In addition, the camera internal parameters obtained with the self-calibration on the 3D test field (Table 4) are comparable to those obtained with the black and white checkerboard calibration session.

\begin{tabular}{|l|c|}
\hline Parameters & Values \\
\hline & pixel \\
$f x$ & 1055.29 \\
$f y$ & 1054.22 \\
$c x$ & 588.433 \\
$c y$ & 356.855 \\
skew & -0.392998 \\
$K_{1}$ & -0.480536 \\
$K_{2}$ & 0.350563 \\
$K_{3}$ & -0.288731 \\
$P_{1}$ & 0.258073 \\
$P_{2}$ & 0.00080406 \\
\hline
\end{tabular}

Table 4. Action camera calibration parameters on 3D test field

\subsection{Chromatic aberration}

Chromatic Aberrations (CA) are caused by the light that follows different paths through the lens system based on each wave length composing it. The two main types of chromatic aberrations are the longitudinal (or axial) chromatic aberration (LCA) and transversal (or oblique) chromatic aberration (TCA) (Fiete, 2004).

The longitudinal aberration occurs when different wavelengths of light are focused at different focal distances, or better at different points on the optical axis. A consequence of this aberration is a degraded focusing quality (Luhmann et al., 2006) that are difficult to reduce. This type of aberration occurs especially in wide-angle lenses (Pöntinen, 2012). The lateral aberration occurs when different wavelengths are focused with different magnifications on the same focal plane and can be corrected in post-processing steps (Cronk et al., 2006). The issue related to the last aberration is investigated by analysing the behaviour of the distortion profiles in separated RGB colour channels. In order to achieve this goal, the calibration session on the flat chessboard was performed separately for each 
individual channels (Red, Green, Blue) of the image set: by using the equations of Brown, camera calibration parameters and the radial distortion profile were generated separately for the three (RGB) channels. The Figure 4 shows the distortion profiles for each channel: it can be noticed that blue channel has a lower distortion than the green one; however, this difference is in the order of just a few pixels.

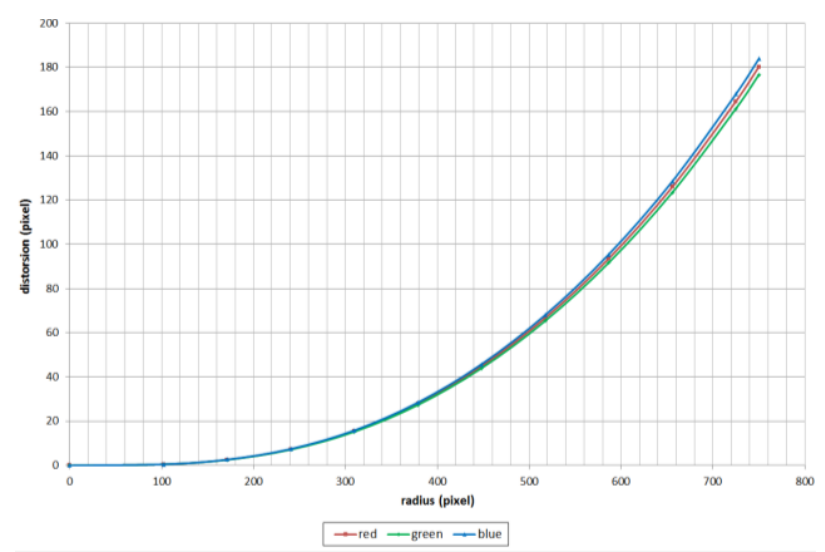

Figure 4. Radial distortion profiles separated for RGB channels

Another way to verify and evaluate the presence of chromatic aberrations is to employ a specific black and white test patterns. Because these charts are made black and white with no other colours, the presence of lens chromatic aberration can be easily identified by the presence of other colours in the image of the pattern itself. The investigation was conducted by employing a Siemens star chart consisting of an even number of tapered wedges pointing to a common centre. As can be seen from Figure 5, by approaching toward the centre of the star, the presence of more colours (chromatic aberration) is visible.
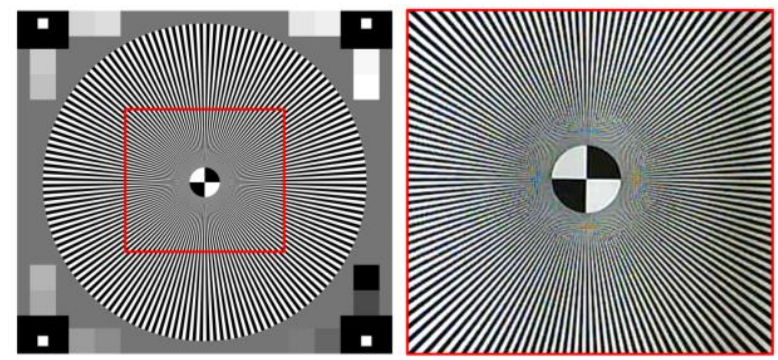

Figure 5. Effect of chromatic aberration on Siemens star

\section{INVESTIGATIONS ON THE TECHNICAL PROPERTIES OF THE ACTION CAMERA}

\subsection{Archaeological site}

The archaeological excavation site for the construction of the new line 1 and line 6 underground station "Municipio" in Naples (Italy) was used as test area for the following tests. Because this construction site insists on a critical area of the historical centre of Naples for a massive presence of ancient artefacts and structures below the actual planking level, a deep archaeological investigation has been preventively required in order to proceed with the infrastructure construction. In such particular cases, the availability of a camera that is portable and usable in different environment conditions, such an action camera is, could be a resolving condition for the need to conjugate the archaeological documentation with the excavation time reduction (by speeding up the artefacts surveys) or with necessity to document a small and/or unexpected evidence in a few minutes.

In the present paper, an attention will be focused on some particular archaeological finds, on which a survey test with an action cam has been performed and the results compared with a DSLR professional camera. The entire operation of image alignment, processing and dense point cloud extraction has been performed with Agisoft Photoscan Professional, while the comparisons between point cloud models has been computed with the open source Cloud Compare software.

\subsection{Survey of the antique naval anchor}

The first test was performed on an antique naval anchor made of wood, found about 6 meters below the actual see level and well conserved (Figure 7). In order to make a comparison with a professional camera generally used for close-range photogrammetry purposes, the survey has been performed both with the tested action camera as well as a professional Canon 5D Mark II DSLR camera with a $24 \mathrm{~mm}$ fixed-lens.

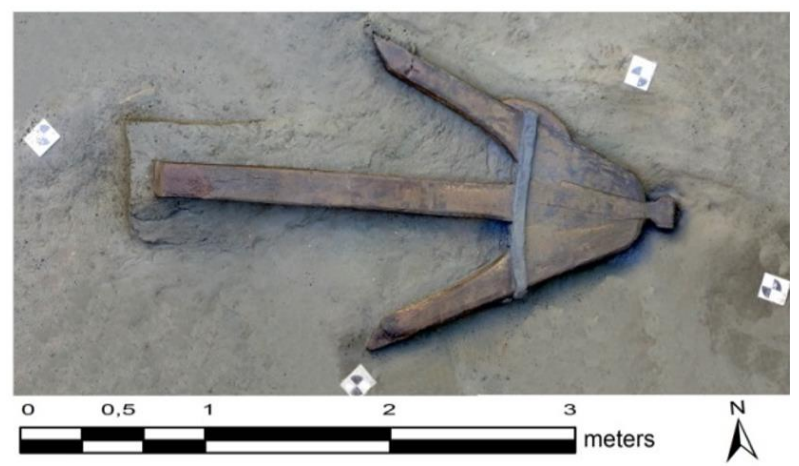

Figure 6. Ortophoto of the anchor

The Table 5 resumes the errors obtained on the GCPs in relation to their absolute coordinates measured with a Total Station on a local reference system: the values obtained with the tested camera are sensibly higher than the one obtained with the professional DSLR one.

\begin{tabular}{|l|c|c|c|c|c|}
\hline $\begin{array}{l}\text { GCP } \\
\text { Name }\end{array}$ & $\mathrm{X}$ & $\mathrm{Y}$ & $\mathrm{Z}$ & $\begin{array}{c}\text { Error } \\
\text { (Canon) }\end{array}$ & $\begin{array}{c}\text { Error } \\
\text { (Action Cam) }\end{array}$ \\
\hline M22 & 7162.209 & 1196.632 & -6.083 & 0.0005 & 0.004 \\
M23 & 7159.548 & 1197.985 & -5.983 & 0.0008 & 0.003 \\
M30 & 7160.244 & 1196.154 & -5.976 & 0.0013 & 0.005 \\
M36 & 7162.237 & 1195.436 & -6.111 & 0.0034 & 0.005 \\
\hline \multicolumn{7}{|r|}{} & $R M S$ & 0.0019 & 0.0043 \\
\hline
\end{tabular}

Table 5. GCPs Coordinates and residuals comparisons

Anyhow, small cameras like the one under exam don't permit to personalize settings as a professional one can do: this means that parameters like ISO, Aperture or Shutter are totally managed by the camera software, and the impossibility to manually manage them and can lead to poor image results in specific cases. This restriction leads to partially blurred images, mainly due to a reduced depth of field, especially at the border of the images: this let the image processing algorithm detects several false points during the image alignment, with a degradation of the final result as a consequence. In order to try to contain this issue, the images have been reprocessed by applying a mask on the border and so reducing the useful image portion to analyse. 


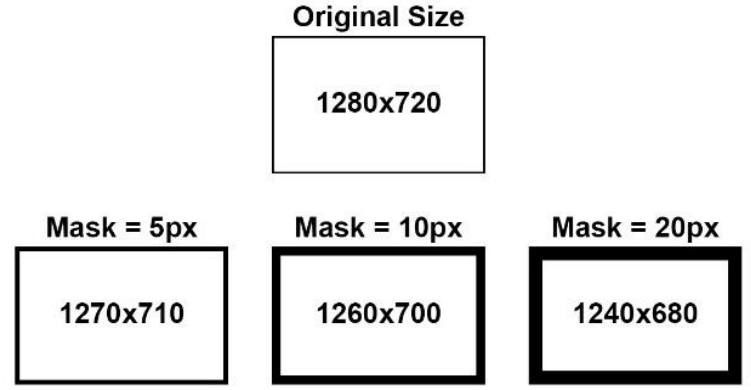

Figure 7. Image masks application and related image size reduction

For the present test, three masks sizes were applied on the images in order to improve the image alignment results.

The Figure 7 shows the original size image (above) and the outcome image dimension reduction (below) after the application of masks which size are $5 \mathrm{px}, 10 \mathrm{px}$ and $20 \mathrm{px}$, that means a reduction of the useful image portion of $2 \%, 4.3 \%$ and $8.5 \%$.

\begin{tabular}{|c|c|c|c|c|}
\hline $\begin{array}{l}\text { GCP } \\
\text { Name }\end{array}$ & $\begin{array}{c}\text { Error } \\
\text { No mask }\end{array}$ & $\begin{array}{c}\text { Error } \\
\text { Mask=5 } \\
\text { pixel }\end{array}$ & $\begin{array}{c}\text { Error } \\
\text { Mask=10 } \\
\text { pixel }\end{array}$ & $\begin{array}{c}\text { Error } \\
\text { Mask=20 } \\
\text { pixel }\end{array}$ \\
\hline M22 & $\begin{array}{c}\mathrm{m} \\
0.004\end{array}$ & $\begin{array}{c}\mathrm{m} \\
0.004\end{array}$ & $\begin{array}{c}\mathrm{m} \\
0.003\end{array}$ & $\begin{array}{c}\mathrm{m} \\
0.002\end{array}$ \\
\hline M23 & 0.003 & 0.003 & 0.002 & 0.001 \\
\hline M30 & 0.005 & 0.005 & 0.004 & 0.001 \\
\hline M36 & 0.005 & 0.004 & 0.003 & 0.002 \\
\hline$R M S$ & 0.0043 & 0.0041 & 0.0031 & 0.0016 \\
\hline
\end{tabular}

Table 6. Residuals of the markers obtained with action camera

After the reprocessing of these three datasets with the applied masks, the results in terms of residuals on the GCP, have been sensibly improved as resumed in Table 6.

In order to evaluate the test-camera performances in relation to the accuracy of the 3D model, among the comparison on the GCPs residuals discussed before, the photogrammetric dense clouds obtained with both cameras have been compared by using Cloud Compare software.

The point cloud obtained with the action camera generally appears quite noisy: the most significant difference of distance between the two point clouds is of about $3 \mathrm{~cm}$, with the $91 \%$ of the points located at a distance less than $1 \mathrm{~cm}$.

In order to improve the accuracy of the point clouds, a "cleaning" operation to identify and exclude outlier points that can negatively influence the results, was performed. This activity was conducted using by using the 'S.Q.R. filter' implemented within Cloud Compare: the filter computes the average distance of each point to its neighbour and then it rejects the points which distance exceeds the average distance plus a number of times the standard deviation value.

Once the filter was applied, about $92 \%$ of the points shows an error between 0 and $8 \mathrm{~mm}$ (Figure 8).

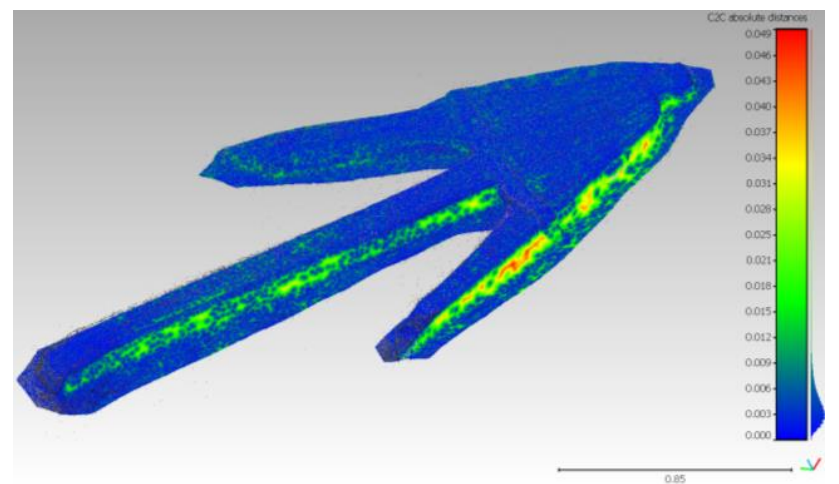

Figure 8. Cloud-to-cloud distance after filtering.

The Figure 8 shows a few points whose distance from the reference cloud is particularly high: this problem occurred on a side of the anchor, due to a very poor illumination of the scene that lead to an increase of matching error on this area during the dense cloud processing.

\subsection{Survey of dredging tracks}

The same methodology above discussed was applied to another particular archaeological discovery: a track belonging to the rests of dredging operations on the tuff rock stratum found at 7 meters circa below the sea level.

This area of interest was surveyed using the low-cost action camera sensor. In particular, the survey performed with the action camera leads to a dataset of 425 images: even in this case, the processing was performed on the original images as well as on masked images, by choosing the 20px size mask (see Figure 7).

\begin{tabular}{|l|c|c|c|c|}
\hline $\begin{array}{l}\text { GCP } \\
\text { Name }\end{array}$ & $\begin{array}{c}\text { Error } \\
\text { No mask }\end{array}$ & $\begin{array}{c}\text { Error } \\
\text { Mask=5 } \\
\text { pixel }\end{array}$ & $\begin{array}{c}\text { Error } \\
\text { Mask=10 } \\
\text { pixel }\end{array}$ & $\begin{array}{c}\text { Error } \\
\text { Mask=20 } \\
\text { pixel }\end{array}$ \\
\hline M21 & $\mathrm{m}$ & $\mathrm{m}$ & $\mathrm{m}$ & $\mathrm{m}$ \\
M30 & 0.021 & 0.018 & 0.001 & 0.018 \\
M34 & 0.019 & 0.013 & 0.009 & 0.013 \\
M40 & 0.010 & 0.017 & 0.011 & 0.017 \\
\hline$R M S$ & 0.017 & 0.014 & 0.010 & 0.0014 \\
\hline
\end{tabular}

Table 7. Residuals of the markers obtained with action camera

Even in this case study, the exclusion of the blurred part of the images bring to a reduction of the RMS on the GCPs as resumed in the Table 7.

The same process alignment processing of the 72 images obtained with Canon camera allowed to obtain accuracies of about $2 \mathrm{~mm}$. The comparison between the action camera points clouds and the reference one shows that the $80 \%$ of the points has a maximum error in $8 \mathrm{~mm}$ and the $20 \%$ has an error to below $1 \mathrm{~mm}$ (Figure 9).

Once obtained the point cloud, it was possible to realize the Dense Digital Surface Model (DDSM) of the area of interest, as shown in the Figure 10. 


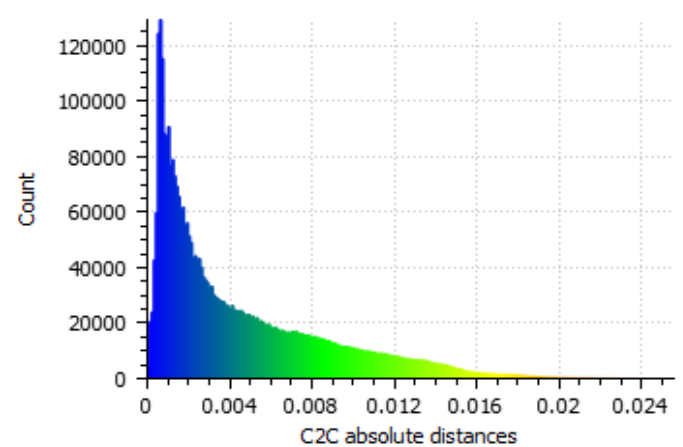

Figure 9. Histogram of distances between the point clouds

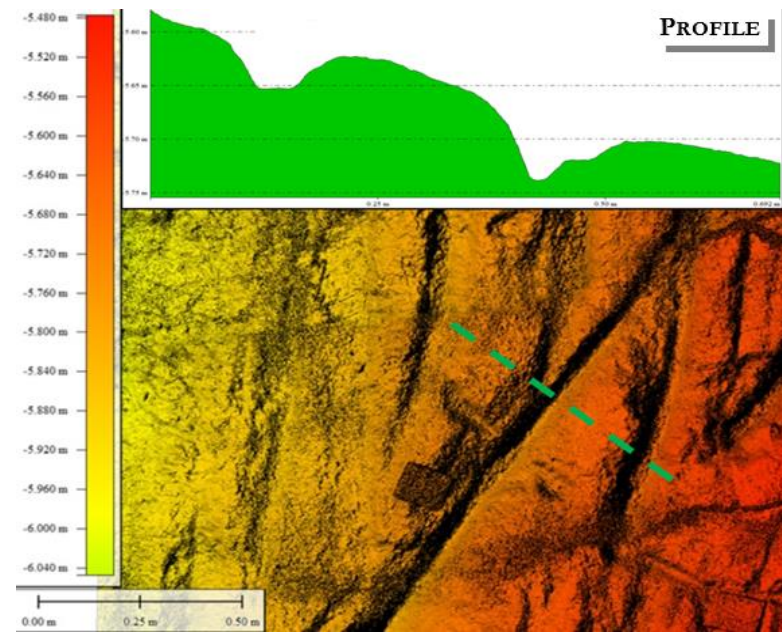

Figure 10. Digital Surface Model

\subsection{Survey of a part of historical wall in narrowed environment}

The present third case shows an extreme survey condition where a small wide-angle lens could have a key role: the artefact is a wall made with tuff blocks technique and reinforced with weirs on one side. Due to its dimensions and to the position of the surrounding reinforced concrete bulkheads, the 3D point cloud has been obtained by merging the photogrammetric and laser scanner techniques. However, a small part of about 1 square meter was almost completely hidden due to the presence of a scaffolding positioned just 30 $\mathrm{cm}$ above the top of the wall (Figure 11a): this condition created a limited operational space making de facto unusable a DSLR camera, due to its dimension as well as the impossibility to keep the camera enough far according to the minimal focalization distance of the mounted lens.

In this case, using the action camera, which has a very short focalization distance, and thanks to the large FOV of the wide angle lens, it was possible to collect enough images to obtain the missing part with a tolerable accuracy degree. The main point cloud of the wall has been obtained by merging the back and front façades point clouds, registered with a Terrestrial Laser Scanner (TLS) and the top part obtained by processing DSLR images (Figure 11b). A total amount of 510 photos has been collected with the action camera to reconstruct the missing part reported in Figure $11 \mathrm{~b}$, even considering the necessity to collect common areas with the DSLR to relatively georeferenced the two clouds. The last down image in Figure 11 shows the result once the two-point clouds blocks have been merged. Lastly, in order to homogenize the colour between several datasets, a tool developed in MATLAB software was used (Pepe et. al., 2017).

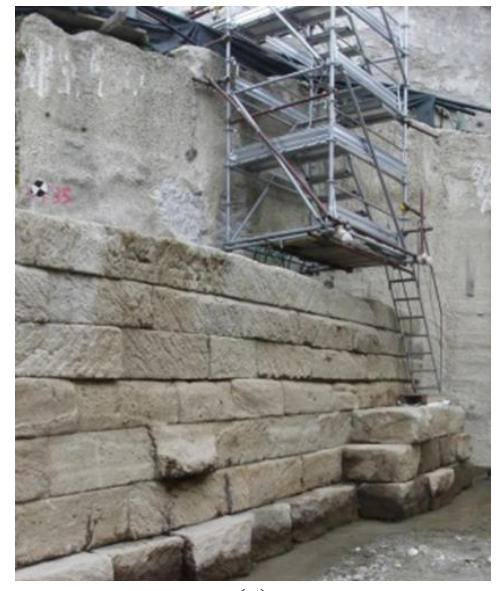

(a)

DSLR IMAGES + TLS

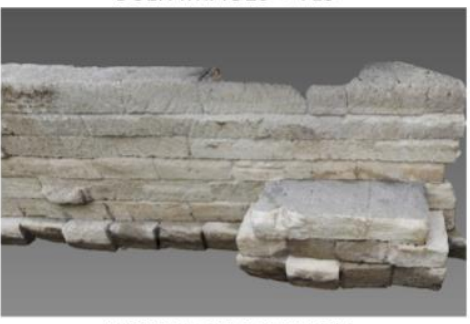

ACTION CAM IMAGES

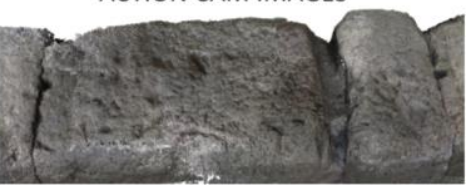

DSLR+TLS+ACTION CAM

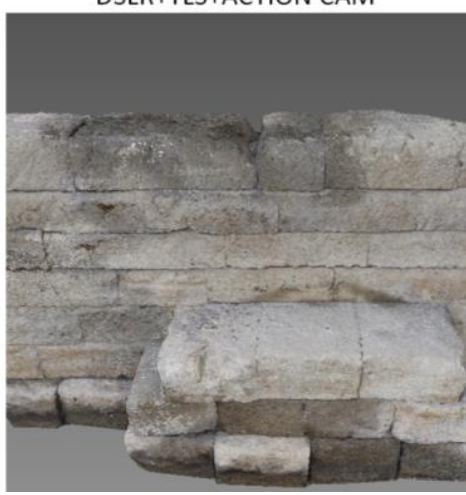

(b)

Figure 11. Panoramic of the study area (a) and point clouds models (b).

\section{CONCLUSIONS}

The tests above described showed that the employment of action camera in cultural heritage field allows to obtain an accuracy of the order of centimetre, in accordance with other tests performed by other research unit on similar topics. The reached accuracy also accomplished the standard specification for image based metric survey contained in "Metric Survey Specifications for Cultural Heritage", in the photogrammetric scale surveys above the scale of 1:50.

Therefore, considering the particular specifics (short focal lens, small size, etc.) of these sensors, they are resulted very useful in photogrammetric applications where space between the object of the survey and the camera is particularly limited. In addition, by partially masking the images and so lightly reduce the FOV, 
that is eliminating the blurred parts of the images, a significant improvement in terms of accuracy has been obtained.

The use of professional DSLR cameras cannot be totally replaced when high accuracy (few millimetres) has to be reached, however, the use of action cameras in certain applications of close range photogrammetry may bring to positive responses.

\section{REFERENCES}

Agisoft Lens Usur Manual, 2017, (last accessed 10/12/2017) http://downloads.agisoft.ru/lens/doc/en/lens.pdf

Balletti, C., Guerra, F., Tsioukas, V., and Vernier, P., 2014. "Calibration of Action Cameras for Photogrammetric Purposes," Sensors, 14(9), pp. 17471-17490.

Brown, D. C., 1971. "Close-range camera calibration, Photogrammetric Engineering," Vol. 37, No. 8, pp.855-866.

Cronk, S., Fraser, M., and Hanley, M., 2006. "Automated metric calibration of colour digital cameras," The photogrammetric record, 21(116), 355-372.

Fassi, F., Fregonese, L., Ackermann, S., and De Troia, V., 2013. "Comparison between laser scanning and automated $3 \mathrm{~d}$ modelling techniques to reconstruct complex and extensive cultural heritage areas," . Int. Arch. Photogramm. Remote Sens. Spatial Inf. Sci., XL-5/W1, 73-80.

Fiete, R. D., 2004. "Lens aberrations", In: Manual of Photogrammetry, Fifth edition, Section 4.1.6, J.Chris Mc Glone (ed.), American Society of Photogrammetry and Remote Sensing (ASPRS), ISBN 1-57083-071-1, pp. 346-349.

Fraser, C. S., 1997. "Digital camera self-calibration", ISPRS Journal of Photogrammetry and Remote sensing, 52(4), 149159.

Hastedt, H., Ekkel, T., and Luhmann, T., 2016. "Evaluation of the Quality of Action Cameras With Wide-Angle Lenses in Uav Photogrammetry," The International Archives of Photogrammetry, Remote Sensing and Spatial Information Sciences, 41, 851.

Jordá, F., Navarro, S., Pérez, A., Cachero, R., López, D., and Lerma, J.L., 2011. "Close range photogrammetry and terrestrial laser scanning: high resolution texturized $3 \mathrm{~d}$ model of the chapel of the kings in the Palencia Cathedral as a case study." In CIPA heritage documentation: Past Symposia-Digital proceedings.

Jurjević, L., and Gašparović, M., 2017. “3D Data Acquisition Based on OpenCV for Close-range Photogrammetry Applications", The International Archives of Photogrammetry, Remote Sensing and Spatial Information Sciences, 42, 377.

Kersten, T. P., and Lindstaedt, M., 2012. "Potential of automatic 3D object reconstruction from multiple images for applications in architecture, cultural heritage and archaeology", International Journal of Heritage in the Digital Era, 1(3), 399-420.

Kim, J. H., Pyeon, M. W., Eo, Y. D., and Jang, I.W., 2014. “An Experiment of Three-Dimensional Point Clouds Using GoPro", Screen, 2704(1440).
Koch, M., and Kaehler, M., March 2009. "Combining 3D laserScanning and close-range Photogrammetry-An approach to Exploit the Strength of Both methods". In Making History Interactive. Computer Applications and Quantitative Methods in Archeology Conference, pp. 22-26.

Luhmann, T., Hastedt, M., and Tecklenburg, M., 2006. "Modelling of chromatic aberration for high precision photogrammetry," In Commission V Symp. on Image Engineering and Vision Metrology, Proc. ISPRS Vol. 36, No. 5, pp. 173-178.

Menna, F., and Troisi, S., 2010. "Low cost reverse engineering techniques for 3D modelling of propellers". In: International Archives of the Photogrammetry, Remote Sensing and Spatial Information Sciences 38 (Part B5), pp. 452-457.

Pepe M., and Parente, C., 2017. "Cultural Heritage Documentation in SIS Environment: an application for "Porta Sirena" in the archaeological site of Paestum", International Archives of the Photogrammetry, Remote Sensing \& Spatial Information Sciences, 42.

Remondino, Fabio, Del Pizzo, Silvio, Kersten, Thomas, Troisi, Salvatore. 2012. "Low-Cost and Open-Source Solutions for Automated Image Orientation-A Critical Overview" in EuroMed 2012, Progress In Cultural Heritage Preservation, LNCS 7616, Springer-Verlag Berlin Heidelberg, pp. 40-54.

Pepe, M., Ackermann, S., Fregonese, L., and Achille, C., 2017. "New Perspectives of Point Clouds Color Management-the Development of Tool in Matlab for Applications in Cultural Heritage", ISPRS-International Archives of the Photogrammetry, Remote Sensing and Spatial Information Sciences, 567-571.

Pepe, M., Fregonese, L., and Scaioni., M., 2018. "Planning airborne photogrammetry and remote-sensing missions with modern platforms and sensors", European Journal of Remote Sensing, 51(1), pp. 412-435.

Pöntinen, P., 2012. "Study on chromatic aberration of two fisheye lenses", In International Society for Photogrammetry and Remote Sensing, Vol. 37, p. 27.

Remondino, F., Fraser, C., 2006. "Digital Camera Calibration Methods: Considerations and Comparisons", In: Proceedings of the International Archives of Photogrammetry, Remote Sensing and the Spatial Sciences, Dresden, Germany, 25-27 September 2006, pp. 266-272.

Stylianidis, E., Patias, P.,Tsioukas,V., Sechidis, L., and Georgiadis, C., 2003. "A digital close-range photogrammetric technique for monitoring slope", In Proc. 11th FIG Symposium on Deformation Measurements Santorini Greece.

Teo, T., 2015. "Video-Based Point Cloud Generation Using Multiple Action Cameras," The International Archives of Photogrammetry, Remote Sensing and Spatial Information Sciences, 40(4), 55.

Westoby, M.J., Brasington, J, Glasser, N. F., Hambrey, M. J., and Reynolds, J. M., 2012. "Structure-fromMotion'photogrammetry: A low-cost, effective tool for geoscience applications," Geomorphology, 179, pp.300-314. 